\title{
Sizes of Superpixels and their Effect on Interactive Segmentation
}

Faculty of Computing \& Informatics

University Malaysia Sabah

Kota Kinabalu, Malaysia

luonggoh@yahoo.com

Soo See, Chai

Faculty of Computer Science and Information Technology

University of Malaysia Sarawak

Kota Samarahan, Malaysia

sschai@unimas.my

\author{
Giap Weng, Ng \\ Faculty of Computing \& Informatics \\ University Malaysia Sabah \\ Kota Kinabalu, Malaysia \\ nggiapweng@ums.edu.my
}

\author{
Muzaffar Hamzah \\ Faculty of Computing \& Informatics \\ University Malaysia Sabah \\ Kota Kinabalu, Malaysia \\ muzaffar@ums.edu.my
}

\begin{abstract}
Semi-automated segmentation, also known as interactive image segmentation, is an algorithm that extracts a region of interest (ROI) from an image based on user input. The said algorithm will be fed the user input information repeatedly until the required region of interest is successfully segmented. Pre-processing steps can be used to speed up the segmentation process while improving the end result. The use of superpixels is one example of such pre-processing step. A superpixel is a group of pixels that share similar characteristics such as texture and colour. Despite the fact that it is used as a pre-processing step in many interactive segmentation algorithms, less studies had been conducted to assess the effects of the size of superpixels required by interactive segmentation algorithms to achieve an optimal result. Therefore, the purpose of this research is to address this issue in order to bridge this research gap. This study will be performed using the Maximum Similarity based region merging (MSRM) with input strokes on selected images from the Berkeleys and Grabcut image data sets, generated by superpixels extractions via energy-driven samples (SEEDS We infer from this research that an image with a minimum of 500 superpixels will aid the interactive segmentation algorithm in producing a decent segmentation result with pixel accuracy of $0.963, \mathrm{~F}$-score of 0.844 , and Jaccard index of 0.756. When the superpixels for an image are raised to 10,000 , the segmentation results degrade. In conclusion, the size of the superpixels would have an impact on the final segmentation results.
\end{abstract}

Keywords - image segmentation, interactive segmentation, input type, strokes, superpixels

\section{INTRODUCTION}

Image segmentation is a crucial part of image processing that allows people to extract a region of interest (ROI) from a picture. There are three forms of image segmentation: manual, semi-automated, and automatic. In image segmentation, fully automated the process should be the ultimate goal. However, due to the intricacy of the images, the output created still faces numerous hurdles [1, 2]. As a result, semi-automated segmentation remains the preferable method.

\section{RELATED STUDIES}

Interactive segmentation is categorized as semiautomatic segmentation. In interactive segmentation, the segmentation system will simultaneously perform segmentation based on the input information from the users until the desired result is obtained. In most cases, the input information consists of a background and a region of interest. In interactive segmentation, different types of user input have been used to provide such information. Strokes, bounding box and seed points are examples of types of user inputs commonly used for interactive segmentation as shown in Fig. 1.

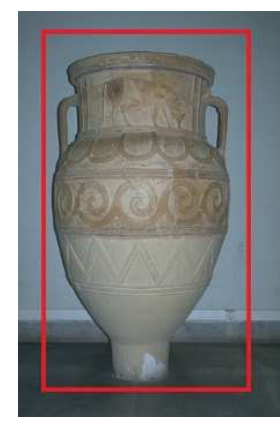

(a)

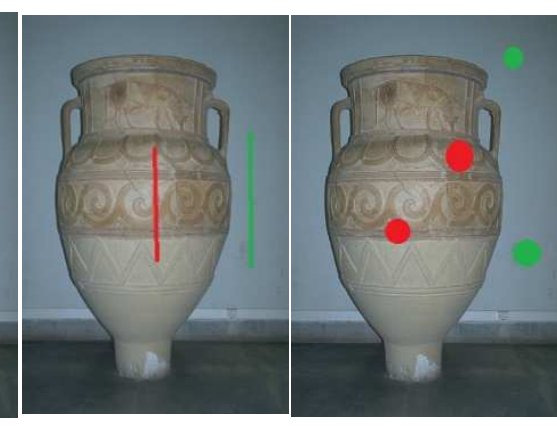

(b) (c)
Fig. 1. Various input types used by users in interactive image segmentation: (a) bounding box (b) strokes (c) seed points.

Once the input information is provided in the segmentation system, the extraction region of interest (ROI) from the background will be executed. Typically, image segmentation is performed on each individual pixel. However, this process consumes a significant amount of processing power and time. As a result, introduction of superpixels assisted in resolving this issue [3]. A superpixel is a group of pixels that share characteristics such as texture or colour. The introduction of superpixels has significantly altered the segmentation process, including the processing steps. Superpixel has been widely used in a variety of applications to date. For example, using remote sensing images for object classification and oil spill detection [4-6] and medical image segmentation [7,8].

Many interaction segmentation methods have used superpixels as one of the pre-processing phases since the debut of superpixels [9-11]. The superpixels algorithms, according to Stutz and Hermans [3], can be classified as watershed, density, graph, contour, path, clustering, energy optimization, and wavelet-based. Fig. 2 shows examples of images created by different superpixels algorithms. However, to our knowledge, up to this date, there is no 